\title{
Alterstice
}

Revue internationale de la recherche interculturelle

International Journal of Intercultural Research

Revista International de la Investigacion Intercultural

\section{Le prix à payer pour devenir sujet de droit : la sélection des réfugiés allosexuels au Canada}

\section{Nathalie Ricard}

Volume 1, numéro 2, 2011

Droits humains et témoignages : l'épreuve de la culture

URI : https://id.erudit.org/iderudit/1077605ar

DOI : https://doi.org/10.7202/1077605ar

Aller au sommaire du numéro

Éditeur(s)

Alterstice

ISSN

1923-919X (numérique)

Découvrir la revue

Citer cet article

Ricard, N. (2011). Le prix à payer pour devenir sujet de droit : la sélection des réfugiés allosexuels au Canada. Alterstice, 1(2), 79-95.

https://doi.org/10.7202/1077605ar

\section{Résumé de l'article}

Depuis les années 1990, le Canada accepte des réfugiés persécutés en raison de leur orientation sexuelle. Le statut de réfugié est octroyé par les membres de la Commission de l'immigration et du statut de réfugié qui évaluent la preuve de l'orientation sexuelle et de la persécution des personnes demandant asile. Or comment prouve-t-on sa sexualité et sa victimisation lorsque l'on a cultivé le silence au sujet de son identité, par peur des représailles, et que le pays d'où l'on vient ne produit pas de documents au sujet de la violence homophobe ? Jusqu'à présent, l'anthropologie ne s'était pas intéressée à la question des réfugiés issus des minorités sexuelles. De leur côté, plusieurs juristes ont cherché à savoir si l'accès à l'asile était équitable pour ces personnes. Les chercheurs se sont peu attardés, toutefois, au langage du droit que ces personnes sont obligées d'utiliser pour se faire reconnaître. Cet article explore les enjeux soulevés par le témoignage des demandeurs et demandeuses d'asile issus des minorités sexuelles, en portant une attention particulière aux exigences du discours juridique qui tend à réifier les dimensions culturelles et sexuelles de la subjectivité. Ainsi, les difficultés de dire la violence ne relèveraient pas uniquement des effets traumatiques de cette dernière, mais aussi du régime d’intelligibilité de la personne qui reçoit le témoignage. Les fonctionnaires canadiens gagneraient à être sensibilisés aux différences culturelles et aux rouages de l'homophobie, à ceux de leur pays comme à ceux dans d'autres sociétés, afin que le processus asilaire soit plus équitable. 


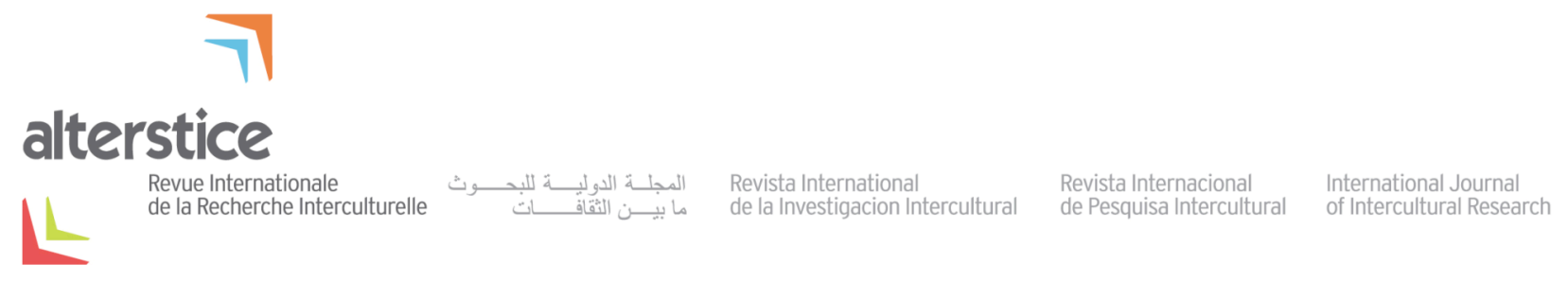

ARTICLE THÉMATIQUE

\section{Le prix à payer pour devenir sujet de droit : la sélection des réfugiés allosexuels au Canada}

Nathalie Ricard ${ }^{1}$

\section{Résumé}

Depuis les années 1990, le Canada accepte des réfugiés persécutés en raison de leur orientation sexuelle. Le statut de réfugié est octroyé par les membres de la Commission de l'immigration et du statut de réfugié qui évaluent la preuve de l'orientation sexuelle et de la persécution des personnes demandant asile. Or comment prouve-t-on sa sexualité et sa victimisation lorsque l'on a cultivé le silence au sujet de son identité, par peur des représailles, et que le pays d'où l'on vient ne produit pas de documents au sujet de la violence homophobe? Jusqu'à présent, l'anthropologie ne s'était pas intéressée à la question des réfugiés issus des minorités sexuelles. De leur côté, plusieurs juristes ont cherché à savoir si l'accès à l'asile était équitable pour ces personnes. Les chercheurs se sont peu attardés, toutefois, au langage du droit que ces personnes sont obligées d'utiliser pour se faire reconnaître. Cet article explore les enjeux soulevés par le témoignage des demandeurs et demandeuses d'asile issus des minorités sexuelles, en portant une attention particulière aux exigences du discours juridique qui tend à réifier les dimensions culturelles et sexuelles de la subjectivité. Ainsi, les difficultés de dire la violence ne relèveraient pas uniquement des effets traumatiques de cette dernière, mais aussi du régime d'intelligibilité de la personne qui reçoit le témoignage. Les fonctionnaires canadiens gagneraient à être sensibilisés aux différences culturelles et aux rouages de l'homophobie, à ceux de leur pays comme à ceux dans d'autres sociétés, afin que le processus asilaire soit plus équitable.

\section{Rattachement de l'auteure \\ ${ }^{1}$ Département d'anthropologie et Centre interuniversitaire d'Études sur les Lettres, les Arts et les Traditions (CÉLAT), Université Laval, Québec, Canada}

\section{Correspondance}

nathalie.ricard.1@ulaval.ca

\section{Mots clés}

Réfugié; minorités sexuelles; droits; témoignage; Canada

\section{Pour citer cet article :}

Ricard, N. (2011). Le prix à payer pour devenir sujet de droit : la sélection des réfugiés allosexuels au Canada. Alterstice, 1(2), 79-96. 


\section{Introduction}

La régulation de la sexualité mise en tandem avec le contrôle de l'immigration servent au modelage de l'État-nation et de la citoyenneté (Luibhéid, 2005). Soixante-seize pays poursuivent toujours les personnes qui ont des relations sexuelles avec un partenaire de même sexe (Bruce-Jones et Itaborahy, 2011). Cinq pays (I'Iran, la Mauritanie, le Soudan, le Yémen et l'Arabie Saoudite), ainsi que certaines régions du Nigéria et de la Somalie, mettent à mort ces personnes. Dans ces États, veiller à la sauvegarde de l'identité nationale et de la dignité humaine implique de signifier clairement que les relations sexuelles entre des personnes de même sexe sont répréhensibles car elles vont à l'encontre de la volonté divine, de l'ordre de la Nature ou du bien-être de la nation. Ailleurs, au contraire, cette réprobation s'inscrit dans le registre des violences et du déni de justice (Renault, 2004). Cette diversité de points de vue et d'attitudes envers les allosexuels ${ }^{1}$ fait en sorte qu'à l'heure actuelle il n'existe pas de metarécit juridique, reconnu par la communauté internationale, qui enchâsse clairement la discrimination liée à l'orientation sexuelle et à l'identité de genre et de sexe ${ }^{2}$. De plus, la décriminalisation sur papier des relations sexuelles entre les personnes de même sexe ne conduit pas nécessairement à des mesures systémiques pour contrer les persécutions ni pour changer les pratiques policières et les mentalités à l'endroit des minorités sexuelles.

Cet article a pour but d'explorer quelques-unes des catégories mobilisées par le témoignage des personnes qui demandent l'asile au Canada en raison de leur orientation sexuelle ou de leur identité de genre. Une interrogation générale sur la construction du témoignage et sur les conditions de sa réception émerge de cette exploration. La Commission de l'Immigration et du Statut de Réfugié (CISR) est le dispositif quasi-judiciaire chargé d'évaluer les demandes de refuge. Le témoignage est la pierre angulaire de ce tribunal administratif. La rencontre entre l'agent ou l'agente d'immigration et le requérant ou la requérante du statut de réfugié offre un éclairage intéressant sur l'attribution de la citoyenneté. Cette attribution est considérée à la fois comme un statut et comme un processus, tant ces deux aspects font partie du matériau d'une citoyenneté en constante redéfinition pour les personnes migrantes. Or, contrairement à ce qui peut se produire dans un contexte d'intervention psychosociale, humanitaire, sanitaire ou éducative, le contexte d'énonciation et de réceptivité des demandes d'asile ne favorise pas l'établissement d'un dialogue interculturel. Une compréhension mutuelle de la violence homophobe est pourtant incontournable dans la pratique du droit d'asile des minorités sexuelles.

Je commencerai par présenter la définition normative d'une "personne réfugiée " selon les instruments juridiques internationaux. Puis j'aborderai l'« allosexualité » et l'« hétéronormativité ». Je ferai ensuite un retour sur la littérature, en relevant particulièrement celle qui concerne le Canada et les enjeux politiques, économiques, moraux et identitaires associés à l'accueil de personnes allosexuelles. Pour terminer, je m'attarderai plus spécifiquement aux deux épistémies du témoignage identifiées par le philosophe Pascal Engel (2005), l'une reposant sur la preuve ou « évidentialisme » et l'autre, sur la confiance et la certitude morale.

\footnotetext{
${ }^{1}$ «Allosexuel » est la traduction québécoise de queer, un terme anglais qui veut dire étrange (voir le Grand dictionnaire terminologique de l'Office québécois de la langue française). Quoique l'anglicisme demeure répandu et pratique puisqu'il peut se transformer en verbe, des organismes québécois comme La Coalition jeunesse montréalaise de lutte à l'homophobie et Alterhéros ont traduit le mot queer pour désigner les sexualités non hétérosexuelles. L'« altersexuel » et l'« altersexualité " sont des expressions utilisées en France. L'acronyme LGBT pour lesbienne, gai, bisexuel(le) et transsexuel(le) ou transgenre est aussi répandu. Je reviens sur la définition du terme dans la section sur la terminologie.

2 En juin 2011, une étape a cependant été franchie vers l'adoption d'un cadre de référence international dans ce domaine. Le Conseil des droits de l'homme des Nations Unies a voté une résolution sans précédent dans laquelle sont rappelés les caractéristiques des droits de l'homme, le rejet des distinctions fondées sur le sexe, de même que ses préoccupations face aux actes de violence et de discrimination commis en raison de l'orientation sexuelle et de l'identité de genre. Pour le texte sur la Résolution A/HRC/17/L.9/Rev.1, voir : Conseil des droits de l'homme des Nations Unies (2011).
} 


\section{Terminologie}

\section{De qui parle-t-on?}

Qu'est-ce qu'une personne réfugiée? En 1969, le Canada signait la Convention de Genève sur les réfugiés - que j'appellerai dorénavant la Convention - et le Protocole relatif au statut de réfugié de 1967. Ces instruments résultent d'un compromis entre des droits individuels et les droits des États-nations à affirmer leur souveraineté. Cette dernière s'exprime, entre autres, par le contrôle des frontières et la sélection des personnes pouvant résider dans le pays. Une personne réfugiée est « une personne qui craint avec raison d'être persécutée du fait de sa race, de sa religion, de sa nationalité, de son appartenance à un groupe social ou de ses opinions politiques " (Loi canadienne sur l'immigration et la protection des réfugiés, 2011). Le requérant ou la requérante doit être reconnu comme ne pouvant pas réclamer la protection du ou des pays dont il a la nationalité. Les menaces de sévices ou la violence doivent être dirigées vers la personne, et exister partout dans le pays. De plus, elles ne peuvent pas résulter de l'incapacité du pays à fournir des soins médicaux ou de santé adéquats.

Les menaces de persécution en lien avec le sexe et le genre ne sont pas explicitées dans la définition-cadre de la Convention. Les femmes et les allosexuel(le)s qui désirent un statut de réfugié doivent le faire sur la base de leur appartenance à un groupe social. Bien que les femmes ne soient pas numériquement un groupe minoritaire, elles demeurent minorisées dans plusieurs sociétés. Le Haut Commissariat des Nations Unies pour les réfugiés (HCNUR, 2008) précise ainsi que des discriminations « clairement préjudiciables pour la personne concernée, par exemple, des restrictions graves au droit de gagner sa vie, de pratiquer sa religion ou d'accéder aux établissements d'enseignement existants " pourraient être interprétées comme des persécutions (p. 4). L'anthropologue Richard Wilson (1997, p. 135) rappelle toutefois qu'il existe un débat entre les légalistes et les contextualistes, ces derniers, contrairement aux premiers, favorisant l'inclusion d'un matériel plus large de données pour interpréter les violences subies par une personne et les affronts à ses droits. L'extrait ci-dessus suggère, cependant, que le HCNUR encouragerait le recours à l'approche contextuelle, ce que devrait aussi favoriser la CISR pour évaluer les demandes d'asile des allosexuel(le)s. Ce n'est toutefois pas toujours le cas, comme nous le verrons dans la section sur la revue de la littérature.

Les allosexuel(le)s regroupent les gais, les lesbiennes, les transgenres, les travesti(e)s, les transsexuel(le)s, les bisexuel(le)s et les intersexes. II peut aussi être question des hétérosexuel(le)s dont l'identité de genre ne correspond pas à leur sexe. Sous toutes réserves, car le mouvement queer peine à sortir de son américanocentrisme blanc (Brotman et Ryan, 2008; Johnson et Henderson, 2005), les Two-Spirited people, soit des bardaches ou des bi-spirituels(le)s, peuvent aussi être inclus dans cette liste, de même que les hijras indiens (Nanda, 1997) ou pakistanais (Naqvi et Mujtaba, 1997), les mahus polynésiens (Lacombe, 2008) ou les tombois (Blackwood, 1998). Si le terme "allosexuel " a le mérite de réduire l'énumération des minorisé(s)s sexuels, il a le défaut d'en gommer les distinctions qui se déclinent selon le genre, l'ethnicité, la culture et la classe (Boellstorff, 2007). Les allosexuel(le)s partagent, cependant, une sexualité non-hétéronormative, rarement désirée par les dieux. En cela, le queer exprime aussi un parti pris radical (voir Warner, 1994), une résistance au régime de la normalité (Luibhéid, 2005), à son modelage identitaire et moral.

\section{De quoi parle-t-on?}

Qu'entend-t-on par " hétéronormativité »? Dans une société hétéronormative, l'hétérosexualité est la norme, la bonne sexualité, naturelle, morale, pure et propre. Cette sexualité bénéficie de privilèges comme la famille, le mariage, sa représentation dans les manuels scolaires, au grand écran, et son acceptation dans l'armée. Dans la " matrice hétérosexuelle », une expression qu'affectionne Judith Butler (2007), les rôles socio-sexuels sont codifiés de façon à ce que le masculin et le féminin soient distincts mais complémentaires dans leur désir. Dans ce cadre d'intelligibilité qui naturalise l'hétérosexualité, les identités homosexuelles et hétérosexuelles sont mutuellement exclusives, et les discriminations et violences peuvent être tolérées, puisqu'elles agissent comme des dispositifs de la police des genres, destinée à rappeler les individus à l'ordre symbolique hétéronormatif. Barry Adam (1998) rappelle que la notion d'hétéronormativité qui porte sur les normes culturelles, sociales et symboliques est surtout utilisée pour l'analyse du discours et pour critiquer la catégorisation binaire de la sexualité. Le courant queer qui a 
développé cette notion déconstruit l'opposition entre l'homosexualité et l'hétérosexualité et donc la notion d'homophobie (p. 388).

Ce concept d'homophobie, qui a été développé par le psychologue George Weinberg dans les années 1960, désigne la peur ou la haine de l'homosexualité (Welzer-Lang et collab., 1994). Alors qu'à l'origine l'homophobie comportait une connotation individuelle (incluant l'homophobie intériorisée) et psychologisante, la phobie des gais et des lesbiennes (lesbophobie dans ce dernier cas) et la transphobie (lorsqu'il s'agit de la peur du transsexualisme) s'appliquent aussi au domaine social dans le langage courant et même scientifique. Il s'agit ici de comprendre cette " phobie » comme le rejet et le mépris des sexualités qui se déploient en-dehors de l'hétérosexualité. Cette peur peut conduire, néanmoins, à des actes de violence envers les minorités sexuelles. D'un point de vue sociologique, ces agressions, la marginalisation et la stigmatisation des allosexuel(le)s s'expliquent par l'hétérosexisme systémique qui soutient la supériorité de l'hétérosexualité au détriment de l'homosexualité. Malgré une épistémie différente, d'autres variations terminologiques de l'homophobie émergent comme l'«homonégativité », terme utilisé notamment dans les enquêtes qui cherchent à mesurer la violence homophobe (voir Blais et collab., 2011). La peur de l'homosexualité stimule ainsi un ensemble de stratégies institutionnelles, discursives et comportementales opposées à la légitimation de cette homosexualité, c'est-à-dire à une remise en cause de la norme hétérosexuelle (Fassin, 2003, p. 99) ${ }^{3}$.

\section{Combien de réfugiés allosexuels y aurait-il?}

Rehaag (2008, p 71) a documenté qu'en 2004, la moitié des 1351 demandes basées sur l'orientation sexuelle avaient été acceptées, soit un taux comparable à celui de la population générale des réfugiés. Dans son mémoire de maîtrise en géographie, Lidstone (2006) a analysé la performativité des demandes d'asile, principalement celles faites par des hommes gais au Canada. Dans son échantillon, comme dans celui de Rehaag (2008), les lesbiennes comptaient pour environ $19 \%$ des demandes d'asile. Rehaag (2008) précise que les bisexuels, dont $15 \%$ étaient des femmes, composaient $7 \%$ du total des demandes d'asile en 2004 (p. 77). Toutefois, seulement $28 \%$ des hommes bisexuels et $10 \%$ des femmes bisexuelles ont obtenu une réponse positive à leur demande.

La CISR ne compile pas en continu des données sur les demandes de refuge liées à l'orientation sexuelle. Après avoir arrêté pendant quelques années de le faire, elle a recommencé à colliger ces données, pour une raison qui demeure inconnue. Parmi les 231 demandes déposées entre la fin mars 2009 et le 30 septembre 2010, 53 avaient été finalisées. Les demandes provenaient d'abord du Mexique ${ }^{4}$, avec 52 demandes, puis du Nigéria (14) et de StVincent et les Grenadines (15). C'est le bureau de Montréal qui a accepté en proportion le plus de demandes (89\%). Celui de Toronto en a accepté $62 \%$, la moyenne nationale étant de $66 \%$. Le bureau de Vancouver a refusé les trois cas qu'il avait fini de traiter.

\section{Un aperçu de la littérature}

\section{Un système asilaire à double tranchant}

Lionel Cantú $(2005)^{5}$, un pionnier de la recherche sur les réfugiés homosexuels, a formulé l'hypothèse suivante : les relations entre les États-Unis et les pays d'Amérique latine, dans les années 1980 et 1990, ont façonné les lois, procédures et politiques du système asilaire américain (p. 70). De plus, le chercheur a soulevé les incongruités de ce système qui réifie la culture de l'étranger ou de l'étrangère alors que cette dernière est reconfigurée sous l'impulsion de la globalisation. Le sociologue, qui s'était particulièrement intéressé aux réfugiés mexicains gais, était préoccupé par le néocolonialisme du système. Certes, celui-ci procure de la sécurité à quelques individus mais, d'un autre côté, " [...] à travers son processus d'incitation, d'évaluation et de circulation des témoignages

\footnotetext{
${ }^{3}$ Pour une discussion critique des concepts " homophobie ", " hétérosexisme " et " hétéronormativité ", voir Bastien Charlebois (2011). Pour une critique culturelle du concept d'homophobie par des anthropologues, voir Murray (2009).

${ }^{4}$ L'obligation depuis 2009 d'obtenir un visa canadien pour les Mexicaines et Mexicains et pour les Tchèques a eu un impact sur les taux de demandes d'asile. L'Organisation des Nations Unies a rapporté en effet une baisse de $84 \%$ des demandes d'asile en provenance du Mexique (Appleyard, 2011), toutes catégories de demandes confondues.

${ }^{5}$ Il s'agit d'un manuscrit que Cantú (1965-2002) n'a pas terminé avant sa mort prématurée. Luibhéid et Stern l'ont retravaillé pour la publication.
} 
individuels à propos de la persécution et de la souffrance, le système asilaire demeure dans un appareil de gouvernance qui génère des images et des relations racistes et colonialistes [...]. » (p. 69-70) ${ }^{6}$. Ces pratiques affectent les migrants et migrantes qui se retrouvent aux Etats-Unis, tout en laissant blanc comme neige leur pays d'accueil alors qu'il joue un rôle dans la mise en place des conditions oppressives que ces personnes ont fuies.

Jusqu'à présent, personne n'a repris l'hypothèse de Cantú (2005) pour l'appliquer à la situation canadienne. À sa suite, l'on pourrait pourtant se demander comment les relations économiques et politiques entre le Canada et le Mexique affectent le système asilaire canadien. Lidstone (2006) s'est d'ailleurs inspiré de la critique de Cantú sur le système asilaire à double tranchant. Le géographe soutient ainsi que le discours du dispositif asilaire canadien renforce les structures qui établissent la supériorité occidentale, sa domination et son racisme à travers, notamment, ses représentations de l' « ici », qui correspond au Canada et aux autres pays occidentaux, et du « làbas ", c'est-à-dire les pays non occidentaux producteurs de réfugiés (Lidstone, 2006, p. 99). En somme, les requérants et requérantes s'insèrent dans une rhétorique de la libération dans laquelle l'imaginaire du progrès, incarné par le Canada, est conforté. Qui plus est, depuis le 11 septembre 2001, ce clivage entre les pays progressistes et ceux qui maltraitent les minorités sexuelles s'est islamophobisé (voir Jenicek et collab., 2009). Les dissidents sexuels sont ainsi considérés comme des "traîtres à leur nation » par leurs compatriotes en raison de l'impératif discursif de la dénigrer durant leur témoignage à la CISR, " afin d'être sauvés par le Canada " (Lidstone, 2006, p. 124-127).

\section{Les allosexuel(le)s comme nouveau groupe social}

Les chercheurs en sciences sociales qui ont été les plus nombreux à analyser la question de "l'asile allosexuel " (queer asylum) ${ }^{7}$ sont des juristes, à travers des recherches comparatives (Berg et Millbank, 2009; Ramanathan, 1996) ou à travers un questionnement sur la meilleure façon d'inclure l'orientation sexuelle dans les instruments juridiques internationaux (Les Principes de Jogjakarta, 2007; Heinze, 2002; Wintemute, 1995). Des juristes se sont aussi intéressés aux demandes de refuge faites spécifiquement en sol américain (Miller, 2005) et canadien (Rehaag, 2008; LaViolette, 1997). La cause Ward, qui s'est rendue à la Cour suprême du Canada en 1993, a marqué un tournant dans la réception des demandes d'asile fondées sur la persécution liée à l'orientation sexuelle ${ }^{8}$. Le pays a aussi fait figure d'exception en acceptant le premier transsexuel, dès 1994 (Ramanathan, 1996). La plupart des pays occidentaux ont suivi l'exemple du Canada pour inclure le genre et l'orientation sexuelle dans leurs législations sur les réfugiés, les États reconnaissant ainsi que les persécutions ne sont pas uniquement d'origine militaire et gouvernementale.

Bien que la cause Ward n'ait pas abordé l'orientation sexuelle comme telle, en orbiter, le juge Laforest avait indiqué que l'orientation sexuelle était une caractéristique immuable sur laquelle un groupe social pouvait être fondé, répondant ainsi à la définition du réfugié selon la Convention (Rehaag, 2008; LaViolette, 1997, p. 3). Malgré les avancées provoquées par l'arrêt Ward, la juriste LaViolette (1997) a avancé qu'il a fixé l'orientation sexuelle, alors que c'est en raison d'une identité sociale, à laquelle des persécuteurs accordent un statut social et politique inférieur, que les personnes qui recherchent l'asile à cause de leur sexualité devraient être reconnues. LaViolette (1997, p. 21) soutient que le jugement Ward a limité l'étendue de la définition possible d'un groupe social particulier en privilégiant les caractéristiques internes d'un groupe donné. Toutefois, comme le souligne Ramanathan (1996, p. 26), les allusions du juge Laforest aux lois discriminatoires et à la perception du risque de

\footnotetext{
${ }^{6}$ " [...] through its processes for eliciting, evaluating, and recirculating individual's testimonies about persecution and suffering, the asylum system remains part of a governance apparatus that generates racist, colonialist images and relations (...) » (Cantú, 2005, p. 69-70). Soucieux de lutter contre l'image négative de son pays, le consul mexicain de Toronto avait ainsi soutenu avoir rencontré plusieurs hommes mexicains qui mentaient à propos de leur orientation sexuelle (Jimenez, 2004).

${ }^{7}$ Selon Ramanathan (1996, p. 2), l'on parle de queer asylum lorsqu'une personne demande le statut de réfugié parce qu'elle est persécutée en raison de son orientation sexuelle, ou lorsqu'elle est violentée parce qu'on la soupçonne de ne pas être hétérosexuelle.

8 La première demande d'asile fondée sur l'orientation sexuelle a été entendue mais rejetée, aux Pays-Bas, en 1981 (Ramanathan, 1996). En 1991, le cas d'un Argentin homosexuel qui avait été accepté comme réfugié en raison des persécutions qu'il avait subies (viols par les policiers, torture avec des électrochocs, coups) avait fait les manchettes canadiennes. La discussion entourant la définition du groupe social que formeraient les homosexuels s'était alors amorcée.
} 
persécution du requérant ou de la requérante, en lien avec le contexte politique, l'opinion publique et les droits humains ${ }^{9}$, indiquent aussi une ouverture pour intégrer des facteurs extérieurs à la définition du groupe social. ॥ s'agit, en outre, d'éléments sur lesquels s'appuyer pour évaluer s'il y a ou non persécution (Ramanathan, 1996).

La position du Canada face aux minorités sexuelles a stimulé le développement de solidarités communautaires et juridiques envers ces dernières. Se réclamant de l'anthropologie juridique et de la cyber-anthropologie, Valérie Shamash (1998) a fait, dans le cadre de son mémoire, l'étude d'un réseau virtuel voué à la défense des réfugié(e)s issus des minorités sexuelles. Elle a démontré comment ce réseau était le fruit de l'engagement d'avocates et d'avocats montréalais. Le réseau a permis à de potentiels clients et clientes de prendre contact avec eux et d'accéder à de l'information juridique sur la situation des gais et lesbiennes, faisant en sorte que les frontières géopolitiques et culturelles de la communauté allosexuelle de Montréal s'élargissent. Notons aussi le mémoire en travail social d'Edward Lee (2009) sur l'insertion de neufs migrants et migrantes allosexuels à Montréal, sa récente revue de littérature sur l'asile allosexuel au Canada (Lee et Brotman, 2011) et la thèse en psychologie de Sharalyn Jordan (2011) qui porte sur les réfugié(e)s issus des minorités sexuelles à Vancouver.

\section{L'identité sexuelle}

Selon Lidstone (2006), afin que l'identité sexuelle des requérants de statut de réfugié soit jugée authentique, quatre critères sont considérés par les commissaires de la CISR $^{10}$ : I'apparence des demandeurs, leurs propos sur leur intimité sexuelle et sur leur désir d'être en relation avec des personnes du même sexe, leurs relations avec une communauté gaie, et leur vie à l'extérieur du placard. Ces critères sont problématiques pour plusieurs raisons. Premièrement, ils reproduisent des stéréotypes ethnocentriques et sexistes sur l'apparence et sur les comportements des individus. En effet, le témoignage des demandeurs du statut de réfugié a plus de chance d'être reconnu, d'être jugé légitime, s'il correspond aux représentations dominantes de l'identité gaie, qui passe par la sortie du placard dans la culture nord-américaine (Berg et Millbank, 2009), selon le modèle Stonewall de la sexualité masculine gaie, de l'identité et de la libération (Stychin, 2004, p. 954).

Dans de nombreux pays où il n'existe pas de filet de sécurité sociale, il n'y pas non plus de salut en dehors de la famille. Ses relations ouvrent l'accès à l'emploi, aux soins de santé et au soutien économique et social. Qui plus est le mariage confère une notoriété et l'accès à la propriété et à l'héritage. De sorte que des gais et des lesbiennes se marient, ont des enfants et mènent une double vie (Whitaker, 2006). Leur mode de vie ne correspond donc pas à l'image que peuvent entretenir les agents et agentes d'immigration envers l'homosexualité. De plus, en tant que femmes, les lesbiennes et les bisexuelles sont particulièrement assujetties aux lois et traditions discriminatoires et restrictives sur le mariage et sur la famille. Le doute sur la nature vraiment gaie (Lidstone, 2006) de la personne qui demande un statut de réfugié motive des membres de la CISR à lui poser des questions inquisitrices sur ses relations sexuelles sans égard pour son intimité (Berg et Millbank, 2009), ce qui participe à la reproduction d’idées hétéronormatives sur les rôles sexuels. Il arrive aussi que des fonctionnaires, inconscients de la double contrainte exercée sur les demandeurs et demandeuses d'asile allosexuels, blâment ces derniers d'avoir pris des risques en s'exposant (Millbank, 2005), alors que la preuve de leur appartenance à un groupe social discriminé est requise pour obtenir un statut de réfugié.

De plus, les attributs identifiés par Lidstone (2006) suggèrent que l’homosexualité est un fait inné et immuable, alors que ses fondements ne sont toujours pas prouvés de manière scientifique, ni du côté des sciences médicales ou psychologiques ni du côté des sciences sociales. Néanmoins, plusieurs gais et lesbiennes défendent aussi leurs revendications en avançant qu'ils n'ont pas choisi leur orientation sexuelle. Comme le rappelle Yoshino (2000, p. 405), l'inaltérabilité attire la sympathie, puisqu'il est considéré immoral de pénaliser des individus pour des caractéristiques ou pour des événements qui dépassent leur contrôle. Cette croyance s'est exprimée dans la jurisprudence antidiscriminatoire américaine (Yoshino, 2000) et canadienne (Rehaag, 2008). De plus, l'immutabilité de l'orientation sexuelle réconforte parce qu'elle précise la place sociale des uns et des autres.

\footnotetext{
${ }^{9}$ L'usage de cette expression est expliqué dans le texte introductif au numéro (Saillant, 2011).
} 
D'autre part, le sexe et l'orientation sexuelle sont rarement théorisés dans les recherches sur l'asile allosexuel et dans les rapports d'agences internationales sur les droits humains comme ceux d'Amnesty International ou de I'International Lesbian, Gay, Bisexual, Trans and Intersex Association ${ }^{11}$. Le sexe renvoie généralement au biologique, telle une donnée invariable, tandis que le genre est associé au culturel, soit à un élément plus fluide. Cette conception de la sexualité suggère aussi que le sexe précède le genre ${ }^{12}$. De plus, dans les discours sur la nation, le genre sert de marqueur culturel au sexe biologique, tandis que la sexualité est utilisée comme un marqueur culturel du désir (Mayer, 2000, p. 4). La lecture libérale des droits reproduit cette organisation sociale, politique et économique des corps sexués tout en laissant une ouverture aux transformations culturelles.

Selon Lidstone (2006, p. 66), les institutions d'État impliquées dans le processus d'asile reproduisent ce qu'elles entendent par " être gai » ou " être lesbienne ». Les hommes et les femmes bisexuels et les intersexes, soit des personnes aux limites des catégories identitaires, échappent, cependant, à une conception binaire et essentialiste de la sexualité et la déstabilisent ${ }^{13}$. Les requérants et requérants bisexuels ont de la difficulté à convaincre les commissaires de la CISR de l'authenticité de leur orientation sexuelle. Les propos qu'ils tiennent sur leur personne de même que leurs histoires de vie sont tenus pour confus et non crédibles (Rehaag, 2008). En revanche, les propos d'une personne transsexuelle qui cherche à établir une correspondance entre son sexe physique et son genre sont jugés comme étant plus plausibles ${ }^{14}$. Les femmes bisexuelles, plus souvent refusées que leurs équivalents masculins (Rehaag, 2008), et les lesbiennes doivent, quant à elles, faire face aux discriminations fondées sur le sexisme, sur le racisme et sur la classe. À cet égard, Amnesty International (2008) rappelle aux activistes des droits de la personne d'utiliser une analyse intersectionnelle pour aborder la situation des lesbiennes. L'organisme souligne que la décriminalisation et l'abrogation des lois qui sont plus souvent utilisées contre les hommes homosexuels que les femmes homosexuelles reçoivent, en comparaison, plus d'attention militante que les politiques, les lois et les us et coutumes qui affectent l'autonomie sexuelle des femmes.

\section{L'alliance médico-légale}

Les travaux de McGhee (2000; 2003) sur les demandes d'asile des homosexuel(le)s en Grande-Bretagne suggèrent que les agents et agentes d'immigration chargés d'évaluer les demandes ainsi que les avocates et avocats impliqués dans le processus se concentrent sur la recherche et sur la production d'une « subjectivité légale " (legal subjectivity). Cette dernière est produite par des technologies juridiques visant à maintenir leur autorité. Elles valident ou invalident l'identité du requérant ou de la requérante, son témoignage ainsi que ses connaissances sur lui-même ou sur elle-même. Le discours médical agit aussi dans le registre de l'expertise. McGhee (2000) a ainsi relevé son utilisation par le pouvoir juridique dans son étude de cas d'loan Vraciu, un Roumain qui avait tenté d’immigrer en Grande-Bretagne au début des années 1990. Lors de son témoignage, Vraciu ne s'était pas présenté en tant qu'homme gai, étant donné qu'il ne s'identifiait pas ainsi, mais il avait allégué avoir été victime de violences à cause de son orientation sexuelle.

Selon Hastrup (2003a, p. 316), le langage juridique est un langage de spécialistes qui limite les capacités de représentation et d'expression de soi. L'anthropologue soulève que le langage positiviste et moderniste instrumentalise les dimensions expressives et symboliques de la violence (Hastrup, 2003a et 2003b). Bien que porteurs de subjectivité, la richesse des pratiques et les silences causés par la violence disparaissent sous la réification des identités produites au nom de la justice. Le conformisme structurel, engendré et maintenu par le

\footnotetext{
${ }^{11}$ Selon Wilson (1997, p. 154), le langage utilisé dans les rapports sur les droits humains reflète celui de l'État-nation moderne. II est utilisé par souci d'efficacité de manière à pouvoir faire changer les politiques d'État. Cet argument pragmatique ne satisfait pas l'anthropologue qui rappelle que les textes des droits humains s'adressent à une communauté hétéroclite d'acteurs sociaux. D'ailleurs, ces derniers ne manquent pas de remettre en question la prétention à l'universalisme des droits humains (Dembour, 2001) et l'importance de les conceptualiser dans leur complexité pour qu'un plus grand nombre de personnes y adhèrent (Muzaffar, 1999).

12 Pour une déconstruction de cet a priori, voir Delphy (1991) dans la perspective du féminisme matérialiste, et Butler (1990) selon la perspective poststructuraliste.

${ }^{13}$ Sur l'effacement épistémique de la bisexualité, voir Yoshino (2000).

${ }^{14}$ La réalité est, cependant, plus complexe. Les transsexuel(le)s ne reproduisent pas tous une orientation hétérosexuelle lors de leur processus de changement de sexe.
} 
champ juridique et par son épistémie, sème ainsi la confusion entre le pouvoir et la vérité (Hastrup, 2003a, p. 317). En outre, lorsqu'il est question de poser un jugement, le processus juridique schématise l'action sociale, de manière à la traduire en faits culturels, et "réduit en squelette " les enjeux moraux pour en extraire des règles $\left(G e e r t z, 2000\right.$, p. 170) ${ }^{15}$. L'obsession de la règle qualifiée de juridisme par Bourdieu (1972), propre à la pensée légaliste chez d'autres (Da Cunha, 2003, p. 12; Hastrup, 2003a et 2003b; Wilson, 1997), perd de vue les principes de justice générateurs de ces règles. Ceux et celles qui n'ont pas accès à cette mise en forme et en mots de leur souffrance risquent de rester en marge du droit.

Afin de saisir la vérité de Vraciu, et pour valider son témoignage, la procureure avait suggéré qu'un examen médical de son anus soit fait. Elle avait justifié sa proposition en rappelant que les femmes qui allèguent avoir été violées doivent subir un examen médical. Sous l'apparence d'une procédure routinière, de surcroît scientifique, la suggestion de la procureure véhiculait, cependant, une idéologie hétérosexiste (McGhee, 2000), représentée de deux façons : l'identité homosexuelle peut se réduire à un acte de sodomie, délibéré ou non, et un homosexuel se reconnaît dans le rôle passif, ou féminin, qu'il prend lors d'une relation sexuelle. L'avocat de Vraciu s'était opposé avec succès à cet examen médical, mais il avait suggéré, en échange, qu'un examen psychiatrique soit conduit. À défaut de soumettre une preuve des traces physiques des violences subies par son client, l'avocat espérait que le témoignage d'un expert sur une psyché traumatisée matérialiserait, à travers un langage factuel et objectif, les conséquences de l'homophobie. Le cas de Vraciu n'est pas isolé. Des évaluations médicales, psychologiques ou psychiatriques accompagnent souvent les dossiers des requérants et requérantes de refuge en quête de crédibilité.

La recherche de Didier Fassin (2004) sur les demandes des migrants pour des titres de séjour en France et sur celles des nationaux, pour le dernier recours, offre une piste pour intégrer l'économie morale à l'analyse du régime asilaire canadien. L'anthropologue avance que les fonctionnaires français ont intégré le discours de l'État sur la pénurie de ressources. Le fonctionnaire doit donc être convaincu de la souffrance du revendicateur pour montrer de la générosité, pour légitimer la demande. Dans le cas des réfugiés, le corps malade est cité à défaut d'une reconnaissance des abus politiques. Alors qu'auparavant les dominés utilisaient leurs corps comme force de travail, ils en viennent à l'utiliser comme source de droits (Fassin, 2004). Ces interactions entre des agents de l'État et les résidents reconfigurent la citoyenneté sur la base de la biolégitimité.

La personne chargée d'évaluer les demandes d'asile au Canada se retrouve dans une situation semblable à celle du fonctionnaire français. Elle doit écouter seule chaque personne et juger si elle mérite la protection canadienne. Les agents et agentes d'immigration s'intéresseraient ainsi autant à la manière dont les violences ont été vécues par le requérant ou la requérante qu'aux violences elles-mêmes. Faute de données sur le contexte de l'agression subie, et d'un témoin de première main qui pourrait la certifier, le rôle des médecins et des professionnel(le)s qui ont le pouvoir de parler du trauma, et de garantir sa vérité, se trouve renforcé. Qui plus est, comme Kleinman et Kleinman (1997) l'ont relevé à propos des victimes de violence politique, le capital culturel associé au syndrome de stress post-traumatique ${ }^{16}$ pourra servir à ces dernières de monnaie d'échange afin d'obtenir de l'assistance morale ou financière. Pour les demandeurs et demandeuses d'asile allosexuel qui recevront une allocation et un accès à des soins de santé, grâce à la Charte des droits et libertés (LaViolette, 1997), le constat de leur victimisation ouvre aussi la porte à la citoyenneté.

Finalement, en plus d'être prises dans une économie politique des rapports Nord-Sud, d'être sujettes aux politiques internes des gouvernements comme à leur économie morale, et d'être confrontées à de possibles biais racistes, hétérosexistes et ethnocentriques de la part des agents et agentes de la CISR, les personnes faisant une demande de statut de réfugié basées sur une discrimination liée à l'orientation sexuelle et à l'identité de genre

\footnotetext{
15 Plus précisément, Clifford Geertz parle de skeletonization des faits dans le processus juridique (Wilson, 1997). Selon Geertz (2000), le langage juridique est une manière spécifique d'imaginer le réel.

${ }^{16}$ La pédopsychiatre Cécile Rousseau (2000) résume ainsi les effets psycho-sociaux et cognitifs du trauma : "À la répétition, mémoire incessante et douloureuse qui s'impose comme un signal d'alarme persistant, comme une blessure qui ne peut guérir, s'oppose l'évitement. Celui-ci peut être total, comme dans la dissociation complète mais, le plus souvent, il est partiel et prend des formes diverses: oubli, évitement plus ou moins conscient de stimuli rappelant le trauma, émoussement émotionnel. Au niveau des liens intimes et familiaux, la distance émotionnelle qui résulte de l'ébranlement des liens fondamentaux de confiance en l'humain est le premier maillon représentant le bris du lien social. » (p. 191).
} 
mettent en jeu une double reconnaissance. La première concerne leur identité en tant que réfugié qui implique, premièrement, d'être reconnu comme une victime qui a besoin de protection et, deuxièmement, que le pays d'origine soit incapable d'offrir cette protection. La seconde reconnaissance concerne l'appartenance à un groupe allosexuel qui implique, premièrement, que la personne soit capable de se reconnaître comme allosexuel(le), deuxièmement, qu'elle ait accès à un tel groupe et, troisièmement, qu'elle puisse le nommer et faire la preuve de cette appartenance à l'étranger ou à l'étrangère qui recueille le témoignage.

\section{Le témoignage des allosexuel(le)s}

\section{Ses épistémies}

Comme le rapporte Engel (2005), le témoignage relève de deux épistémies qui s'opposent : " celle de la preuve ou évidentialisme - on n'est justifié à croire que si l'on dispose de données empiriques fiables - et celle de la confiance et de la certitude morale, selon laquelle certaines croyances basées sur une forme de certitude primitive se passe de preuves » (p. 59). La rationalité du système social décliné dans ses composantes juridiques et médicales relève de la première épistémie et non de la seconde, qui participe du sens commun où « toute assertion est digne de créance tant qu'elle n'a pas été montrée fausse ». Kirmayer (2003) décrit dans les termes suivants l'épistémie des membres de la CISR :

Les membres de la CISR traitent le récit du revendicateur comme un acte théorique de positionnement en vue de réclamer un statut social spécifique. Ils partagent une épistémologie implicite selon laquelle un exposé crédible et véridique est isomorphique, avec une seule séquence d'événements. Une véritable histoire est un compte-rendu fixe, fidèle, et reproductible d'événements historiquement vérifiables. Puisque la séquence précise et détaillée des événements antérieurs à la migration ne sont pas vérifiables en dehors du récit du réfugié, la CISR doit compter sur des rapports de recherche qui fournissent un arrière-plan à des exposés génériques et sur son expérience avec des revendicateurs antérieurs. De ce fait, le récit du revendicateur est suspect avant d'être énoncé. (Kirmayer, 2003, p. 170) ${ }^{17}$

Les difficultés sont donc nombreuses pour les demandeurs et les demandeuses allosexuels qui doivent produire un témoignage, être entendus et crus par la CISR. Premièrement, il se peut qu'il n'y ait pas de documentation spécifique ou de recherches qui portent sur l'homophobie dans les pays qu'ils quittent, de surcroît lorsque la discrimination basée sur l'orientation sexuelle n'est pas reconnue. Deuxièmement, leur témoignage peut contenir des informations contradictoires, comme dans le cas des personnes bisexuelles, ce qui menace la véracité de leur témoignage, en la rendant variable et non reproductible, ce qui fait que le genre d'histoire qu'ils racontent n'est pas jugé véridique et recevable par la CISR.

De plus, comme le rappelle le Rainbow Refugee Committee (2010), les persécutions homophobes, lesbophobes et transphobes sont moins documentées que les autres types de persécutions et les données par pays concernant les violences faites aux allosexuel(le)s sont peu nombreuses et insuffisantes (p.4). L'organisme, qui soutient les réfugiés allosexuels à Vancouver, a aussi fait état de la difficulté qu'avaient les demandeurs et les demandeuses d'asile à révéler des aspects intimes de leur vie, d'autant plus qu'ils avaient été vécus sous le mode du secret (p. 5). Ainsi, se dévoiler, c'est courir le risque, à nouveau, de s'exposer à un déni de reconnaissance, à une nouvelle blessure morale, pour reprendre la terminologie d'Emmanuel Renault (2004).

De sorte que, même si une personne quitte son pays parce que sa sécurité est en jeu, il peut lui paraître inconcevable de dévoiler à l'agent ou à l'agente d'immigration qui enregistre sa déposition, dès son arrivée au Canada, les motifs pour lesquels elle demande un statut de réfugié. Elle a appris toute sa vie à dissimuler son identité intime et à se méfier de la police ou des représentants de l'ordre (Luibhéid, 2002). Mais cette omission peut lui être fatale. La CISR peut rejeter la requête si des contradictions sont relevées entre la déposition initiale et le témoignage rendu ultérieurement, en dépit du fait que ces incohérences s'expliquent par la difficulté à dire les

17 " The members of the IRB treat the claimant's narrative as a theoretical act of positioning designed to claim specific social status. They hold an implicit epistemology in which a credible and truthful account is isomorphic with a single sequence of events. A truthful story is a fixed, reliable, and reproducible account of historically verifiable events. Since the precise sequence and details of events prior to migration are unverifiable outside the refugee's narrative, the IRB must rely on research dossiers that provide the background for generic accounts and their experience with previous claimants. As such, the claimant's account is suspect before it is uttered. " (Kirmayer, 2003, p. 170) 
violences subies, notamment à cause de leur impact traumatisant (Berg et Millbank, 2009; Kirmayer, 2003; Rousseau, 2000; Rousseau et collab., 2002), par homophobie intériorisée ou par ignorance de ses droits.

En second lieu, les allosexuel(le)s doivent faire face à l'épistémie du sens commun hétérosexiste. Cette dernière est en contradiction avec la première, comme le suggère Engel (2005). En effet, les allosexuel(le)s ne peuvent pas tabler sur une commune assertion de leur identité sexuelle. Cette commune assertion, digne de créance, existe, certes, mais dans d'autres registres identitaires comme celui du travail. De sorte que les demandeurs et demandeuses de statut doivent aller contre " la certitude primitive " pour prouver qu'ils ne sont pas hétérosexuels, contrairement aux croyances de l'évidence même. L'identité sexuelle est donc le pivot autour duquel le témoignage doit se construire, les autres dimensions identitaires étant mises de côté du fait de ce discours victimaire recherché par la CISR.

Or l'apparition de la reconnaissance de soi dans le langage juridique confère une puissance d'agir. Dans Le récit de soi, Judith Butler (2007) rappelle que la capacité réflexive à dire la vérité est limitée par ce que le régime d'intelligibilité peut admettre comme dicible. La trame historique d’un État laïc (ou en voie de sécularisation) permet de saisir s'il est possible de dire son homosexualité sans passer pour criminel, fou ou déviant. Les luttes pour la justice sociale ont participé à rendre les discriminations selon le genre et la sexualité moins acceptables et banales. Les normes sociales, culturelles et juridiques ont été transformées grâce à la décriminalisation de I’homosexualité au Canada en 1969, au retrait de l'homosexualité du manuel psychiatrique des diagnostics des maladies mentales en 1973, à la reconnaissance des familles homoparentales au Québec en 2002 et enfin à la légalisation du mariage civil pour les couples de même sexe en 2005. Les membres de la CISR devraient faire abstraction, par professionnalisme, de leurs valeurs personnelles, y compris les valeurs religieuses, pour suivre le discours public qui reflète ces choix de société faits en faveur de l'équité citoyenne. En fait, pour statuer, les fonctionnaires recherchent dans le témoignage des faits en adéquation avec ce que la loi prescrit. La reconnaissance peut donc être comprise comme une forme de pouvoir qui transforme les individus en sujets à partir de ces codes sociaux et culturels. En ce qui concerne le statut de réfugié pour des allosexuel(le)s, la subjectivation a donc comme conséquence politique une citoyenneté renouvelée grâce à la reconnaissance de ce qui est dit sur un soi sexué.

\section{Le face-à-face}

La difficulté à dire une violence qui serait homophobe ne relèverait donc pas uniquement de conséquences psychologiques sur les individus. Elle est aussi déterminée par la capacité des demandeurs et des demandeuses d'asile à la mettre en forme selon les codes sociaux, culturels et juridiques de leurs interlocuteurs ou interlocutrices, de même que par le contexte d'énonciation. Ce contexte est hautement anxiogène car il n'y pas de droit d'appel institué présentement. Moyennant la somme de $2500 \$$, la cour fédérale canadienne peut réviser un cas sur des questions de technicalités judiciaires et non sur le fond de la cause. Un droit d'appel pourrait toutefois être institué, advenant l'adoption par le parlement canadien du projet de loi C-31, soit la Loi modifiant la Loi sur l’immigration et la protection des réfugiés.

Seuls les réfugié(e)s qui proviendraient de pays désignés comme étant sécuritaires pourraient néanmoins se prévaloir de ce droit d'appel. Les modalités de désignation des pays dit "sécuritaires " sont hautement contestées puisque le ministre de la Citoyenneté, de l'Immigration et du Multiculturalisme s'octroierait le pouvoir de décider sans comité consultatif quels pays seraient sécuritaires ${ }^{18}$. Les groupes de défense des réfugié(e)s craignent ainsi que ce soit des motivations politiques et économiques qui prennent le pas sur une évaluation impartiale des conditions du pays en matière d'engagement et de pratiques de protection des droits humains. Par ailleurs, cette

\footnotetext{
${ }^{18}$ Le Conseil canadien pour les réfugiés, l'Association Canadienne des Avocats et Avocates en Droit des Réfugiés, Amnistie internationale, l'Association canadienne des libertés civiles, Solidarité sans frontières, Human Rights Watch, No one is illegal, le Conseil québécois des gais et lesbiennes, des groupes LGBT du Québec comme Action gaie, lesbienne, bisexuelle, transsexuelle avec les réfugié(e)s et immigrant(e)s (AGIR), Au-delà de l'Arc-en-ciel (ADA), Helem Montréal, Gai et lesbiennes asiatiques de Montréal (GLAM), et Arc-en-ciel d'Afrique, de même que le diocèse anglican de Québec unissent leurs voix pour dénoncer le projet de loi C-31. Le représentant des Nations Unies au Canada a aussi émis une réserve sur certaines propositions contenues dans ce projet de loi.
} 
proposition est aussi décriée car elle contrevient à l'esprit de la Convention qui avance que toute personne craignant pour sa sécurité devrait pouvoir réclamer la protection d'un autre État. Les demandes d'asile devraient donc être évaluées au cas par cas et non selon la nationalité des demandeurs et des demandeuses de refuge. Les minorités sexuelles seront particulièrement pénalisées par cette nouvelle mesure, puisqu'il reste de nombreux pays qui, bien qu'ils puissent paraître "sécuritaires " pour d'autres raisons, ne reconnaissent pas que les droits des minorités sexuelles sont des droits humains. De plus, la nouvelle loi réduirait les délais pour soumettre les demandes d'asile, les documents et pour obtenir une audience.

Une partie du travail des avocats et avocates, des conseillers et conseillères en immigration et des activistes devrait consister à préparer les postulants et les postulantes à leur audience, par le rapiéçage de leurs fragments de souvenirs, dispersés par la peur et par le temps, de manière à ce qu'ils puissent s'insérer dans la logique des fonctionnaires, dans le régime d’intelligibilité de ceux et celles qui détiennent le pouvoir de statuer sur leur sort. En effet, le témoignage oral doit corroborrer l'ensemble des documents présentés pour étayer la preuve de la persécution et de l'identité sexuelle du réfugié(e) et son impossiblité à être protégé dans le pays qu'il fuit. De plus, le témoignage oral du demandeur ou de la demandeuse d'asile doit correspondre aux dépositions écrites de I'histoire de vie enregistrées auprès des autorités de l'immigration depuis l'arrivée au Canada ou dans les pays où une demande d'asile aurait été faite antérieurement. Or, comme le rappelait Bola Akinnusi lors d'une conférence donnée à une centaine de réfugié(e)s dans un groupe communautaire, plusieurs ne bénéficient pas d’un tel appui pour préparer leur audience. Selon cette juriste, la clef du succès pour « réussir » cette audience demeure pourtant l'établissement de la crédibilité de l’histoire de la personne et de son identité.

Durant ce moment liminal de redéfinition identitaire, la personne, suspendue à chaque étape du processus de détermination de son statut, recherche des appuis. Elle trouve néanmoins difficilement une communauté d'appartenance. Les demandeurs et demandeuses d'asile sont partagés entre la communauté gaie, majoritairement blanche et francophone, au Québec, ou blanche et anglophone, dans le reste du Canada, et les associations et les lieux de culte qui forment une communauté diversifiée mais également fermée à I'homosexualité $^{19}$. L'altérisation des réfugié(e)s ne relève pas uniquement, cependant, des clivages linguistiques, sexuels et raciaux. La notion même de citoyenneté s'érige comme marqueur, tandis qu'au droit fondamental de pouvoir demander l'asile dans un pays se substitue l'idée qu'il s'agirait d'un privilège. De plus, qu'est-ce qu'un ou une réfugié(e) sinon quelqu'un qui risque de repartir, gaspillant ainsi l'argent des contribuables? Les préjugés à l'endroit des réfugié(e)s comme profiteurs et profiteuses du système ou comme menaces à l'identité nationale et aux valeurs dites canadiennes reviennent avec force (Atak, 2010). Ainsi, d'un côté, la porte du placard pourrait s'entrouvrir tandis que, de l'autre, elle continue à se refermer sur la complexité des subjectivités. Le soutien communautaire s'avère cependant incontournable du point de vue de la survie matérielle, le rassemblement de la preuve pour obtenir un statut de réfugié exigeant de la force morale, du temps et de l'argent. A titre d'exemple, faire venir un document du Nigéria peut coûter $300 \$$, alors que les demandeurs et demandeuses d'asile reçoivent une allocation gouvernementale de moins de $500 \$$ par mois et qu'un permis de travail coûte $150 \$$. La peur de la déportation avec laquelle les réfugié(e)s doivent composer s'ajoute donc à l'insécurité quotidienne de répondre aux besoins élémentaires de nourriture, d’habitation et de vêtements. Les personnes requérant le statut de réfugié au Canada attendent en moyenne deux ans avant d'être fixées sur leur sort (Lacroix, 2004). Des chercheur(e)s ont documenté comment cette attente anxiogène pouvait être une source de revictimisation (Diallo et Lafrenière, 2007).

Pendant que le processus du droit prend corps, un décalage énorme peut exister entre les espérances de la personne requérante, pour qui accéder à la catégorie de réfugié devient une planche de salut, et sa ou son vis-à-vis, pour qui il peut n'être question que d'attribuer une citoyenneté administrative. Rehaag (2010) soulève, cependant, que les modalités structurelles et organisationnelles n'expliquent pas à elles seules la grande variabilité d'acceptation des demandes qui existe au sein de la centaine de membres qui composent la CISR ainsi qu'entre les régions du Canada. Le tableau 1 rend compte de cette diversité.

\footnotetext{
${ }^{19}$ Sur l'insertion des immigrants gais et des immigrantes lesbiennes à Montréal, voir Chamberland et Gagné (2008); à Toronto, voir Smith (2008).
} 
Tableau 1 : Nombre de décisions positives et négatives et taux d'acceptation d'un échantillon de $17 \%$ des membres de la CISR

\begin{tabular}{|c|c|c|c|c|}
\hline Nom des membres & $\begin{array}{l}\text { Nombre de } \\
\text { décisions } \\
\text { négatives }\end{array}$ & $\begin{array}{c}\text { Nombre de } \\
\text { décisions } \\
\text { positives }\end{array}$ & $\begin{array}{c}\text { Nombre total de } \\
\text { décisions }\end{array}$ & $\begin{array}{c}\text { Taux } \\
\text { d'acceptation } \\
(\%)\end{array}$ \\
\hline Berryx, Clifford & & & 206 & 100,00 \\
\hline Bissonnette, Alain & 63 & 52 & 115 & 45,22 \\
\hline Bosveld, Edward & 36 & 50 & 87 & 57,47 \\
\hline Bourque, Renée & 84 & 53 & 139 & 38,13 \\
\hline Bruin, Lucinda & 43 & 18 & 61 & 29,51 \\
\hline Budaci, Stephen & 12 & 4 & 16 & 25,00 \\
\hline Buttigieg, Coralie & 41 & 45 & 86 & 52,33 \\
\hline Byczak, Michel A. & 94 & 16 & 137 & 11,68 \\
\hline Chevrier, Marie & 9 & 16 & & 98,29 \\
\hline Costa, Ana & 14 & 17 & & 63,16 \\
\hline Cousineau, Louis & 31 & 24 & 55 & 43,64 \\
\hline Cryer, Douglas & 64 & 5 & 69 & 7,25 \\
\hline Cyr, Roxane & 34 & 7 & 41 & 17,07 \\
\hline Davis, William & 33 & 98 & 131 & 74,81 \\
\hline Dawson, Richard & 2 & 18 & & 98,85 \\
\hline Dawsonx, Richard & & 3 & & 100,00 \\
\hline Delisle, Ruth & 87 & 28 & 115 & 24,35 \\
\hline Dhir, Rena & 39 & 17 & 56 & 30,36 \\
\hline Dickenson, Kirk & 100 & 32 & & 24,09 \\
\hline
\end{tabular}

Source : adapté et traduit librement de Rehaag (2010)

La performance de Doug Cryer, un ancien directeur des politiques pour le groupe évangélique-pentecôtiste Evangelical Fellowship of Canada nommé à la CISR, se dénote de celle de ses collègues. Le groupe pour lequel il travaillait s'oppose activement à toute forme de reconnaissance pour les gais et les lesbiennes. Comment vous sentiriez-vous en sachant cela de votre évaluateur, lui qui a le pouvoir de décider de votre avenir? Comment Monsieur Cryer reçoit-il la demande d'un requérant gai? Entend-t-il la violence racontée par une requérante allosexuelle qui n'a pas de rapport de police pour attester de l'agression dont elle a été victime, dont le corps ne porte peut-être pas de marques ou dont les marques sur sa psyché demeurent fuyantes?

Comme Lidstone (2006, p. 95) l'a relevé dans sa recherche sur les requérants gais, le demandeur d'asile allosexuel a avantage à se présenter, lors de ses entrevues, comme un « bon » citoyen, c'est-à-dire qu'il doit « se distancier des stéréotypes négatifs sur le sexe gai dans les parcs pour figurer comme un consciencieux consommateur de la business locale gaie ${ }^{20}$. Le retrait de toute allusion aux droits des gais et des lesbiennes d'une brochure gouvernementale, à la suite de l'intervention du bureau du ministre de la Citoyenneté, de l'Immigration et du Multiculturalisme (Nadeau, 2010), peut aussi être considéré comme un indicateur du type d’homosexuel recherché par le gouvernement. La brochure en question est le document qui est remis à tous les immigrants et immigrantes qui veulent obtenir la citoyenneté canadienne afin qu'ils préparent leur examen. II a été publié en novembre 2009 et la seule référence aux homosexuels qui figure dans cette nouvelle édition est une photographie du nageur olympique Mark Tewsbury, présenté comme un important militant des gais et lesbiennes du Canada (Beeby, 2010). Avis aux intéressés : si vous voulez immigrer au Canada et que vous n'êtes pas hétérosexuel, vous avez intérêt à être le plus méritant possible et à avoir l'air le plus correct, voire le plus straight possible, c'est-à-dire blanc, masculin, et de classe moyenne.

20 " [...] to distance [themselves] from the negative stereotypes of gay park sex while figuring as a thoughtful consumer of local gay business » (Lidstone, 2006, p. 95). 
Rousseau et ses collègues (2002) ont habilement décrit tout l'arbitraire que pouvait contenir le rejet des demandes de statut de réfugié : par exemple, ce rejet se fait sans demande de clarification, ou quand les rapports médicaux et psychologiques sont mal compris ${ }^{21}$. L'équipe de Rousseau ne fait pas état, cependant, de réclamations sur la base de la discrimination liée à l'orientation sexuelle. En revanche, celle de Berg et Millbank (2009) a analysé spécifiquement de tels dossiers rejetés par les responsables chargés de statuer sur les réfugiés au Canada, au Royaume-Uni et en Australie. Des similitudes se dégagent des analyses des chercheurs. Elles révèlent d'une part la difficulté à repérer en quoi consiste la preuve, qui dépend souvent du contexte, et d'autre part une insensibilité à l'égard de la souffrance mise à nu. De plus, les fonctionnaires chercheraient à se protéger des témoignages difficiles à supporter et à imaginer, le vécu abordé leur étant souvent complètement étranger, leur apparaissant même parfois hostile. Qui plus est, ils sont généralement mal informés de la situation politique et culturelle du pays d'origine du demandeur ou de la demandeuse, et de la vie des minorités sexuelles de ce pays. Les membres de la CISR gagneraient donc à être sensibilisés aux rouages de l’homophobie, aux leurs comme à ceux d'autres sociétés, ainsi qu'aux différences culturelles et à l'impact de la violence sur la faculté de dire et de se dire.

\section{Conclusion}

Au Canada, l'admission des réfugiés n'est pas une affaire de charité ni d'abus potentiel de la générosité canadienne, mais de respect des obligations internationales signées par le pays, de respect du droit canadien et de respect envers une commune humanité qui s'exprime, notamment, à travers l'application du droit humanitaire et des droits de la personne. L'accueil des réfugiés mobilise une économie morale à travers la reconnaissance de la souffrance et une volonté de l'apaiser, voire d'y mettre un terme (Crépeau et collab., 2009). Comme nous l'avons vu, la CISR jongle avec ces différentes obligations. Il appert, toutefois, qu'un travail de sensibilisation auprès de ses membres et l'intégration de nouvelles connaissances à propos des causes et des conséquences des différentes formes d'homophobie, au Canada et dans les pays d'origine des demandeurs et demandeuses d'asile, pourraient rendre le processus de refuge plus équitable pour les allosexuel(le)s.

La tension entre les approches légalistes et contextualistes dans le champ juridique est fructueuse. Elle permet aux activistes des mouvements sociaux de faire reconnaître leurs revendications identitaires et de redistribution, comme en témoignent les modifications au langage codé du droit. En rajoutant aux motifs possibles de réclamation de refuge l'appartenance à un groupe social, les rédacteurs de la Convention, adoptée en pleine guerre froide, avaient fait preuve de clairvoyance, les réfugiés de demain étant ceux qui rechercheront l'hospitalité des pays qui les toléreront comme minorités. Des individus, autrement invisibles, peuvent ainsi devenir sujets de droit, malgré les limites du langage du droit et le prix à payer. S'ajoutant aux défis de l'immigration et aux souffrances des blessures, ce prix à payer inclut aussi les réifications identitaires, culturelles et morales ainsi que le cautionnement de structures inégalitaires de pouvoir et d'autorité utilisées pour changer les lois et les pratiques.

Cet article illustre la manière dont les témoignages des demandeurs et demandeuses d'asile allosexuel sont façonnés pour répondre aux attentes du système politique et juridico-médical qui intervient dans la régulation de la citoyenneté et de la migration. Des ethnographies portant sur les interactions entre eux et les agentes et agents gouvernementaux documenteraient les silences, les exclusions et les malentendus entourant la violence homophobe et celle du système. L'amélioration des pratiques passe par des recherches sur le processus de demande d'asile des minorités sexuelles au Canada et faisant connaître leur détresse, surtout lorsque cette dernière est exprimée dans un langage non expert et échappant à la contrainte de la juridicité. Les domaines d'intervention interculturelle du travail social, de la psychologie et des soins pourraient aussi bénéficier de l'apport de telles recherches. Enfin, une anthropologie de l'asile allosexuel contribue à introduire la sexualité dans le champ des études sur les réfugiés et à faire re-connaître ces réfugié(e)s qui reconfigurent les frontières du privé et du public et celles du local et du global.

\footnotetext{
${ }^{21}$ Kirmayer (2003) insiste sur la question de l'imaginaire pour évaluer la santé mentale des réfugié(e)s. Ces derniers peuvent ne pas parvenir à établir des ponts entre le monde qu'ils ont quitté et celui dans lequel ils se trouvent maintenant. Mais le psychiatre mentionne aussi la faillite imaginative des cliniciens et cliniciennes qui les empêche de comprendre les situations de vie difficiles des demandeurs et des demandeuses d'asile. Ils n'entrent pas dans l'espace terrifiant qui habite l'autre et, comme les membres de la CISR, ils sont à la recherche de faits tels qu'ils sont décrits dans leurs livres. En outre, ils ne connaissent souvent de la politique et de la culture du pays des réfugié(e)s que ce que l'actualité leur livre.
} 


\section{Références bibliographiques}

Adam, B. (1998). Theorizing homophobia. Sexualities, 1(4), 387-404.

Amnesty International. (2008). Love, hate and the law. Decriminalizing homosexuality. Londres : Amnesty International.

Appleyard, F. (2011, 29 mars). Asylum bids to Canada plummet 30 per cent. The Gazette, Montréal, Québec, p. A1.

Atak, I. (2010). Enjeux pour le système d'asile canadien. Vivre ensemble, 17(58), 3-8.

Bastien Charlebois, J. (2011). Au-delà de la phobie de l'homo : quand le concept d'homophobie porte ombrage à la lutte contre l'hétérosexisme et l'hétéronormativité. Reflets, revue d'intervention sociale et communautaire, 17(1), 112-149.

Beeby, D. (2010, 4 mars). Les droits des gais retirés d'un guide remis aux immigrants. La Presse, p. A8.

Berg, L. et Millbank, J. (2009). Constructing the personal narratives of lesbian, gay and bisexual asylum claimants. Journal of Refugee Studies, 22(2), 195-223.

Blackwood, E. (1998). Tombois in West Sumatra: constructing masculinity and erotic desire. Cultural Anthropology, 14(4), 491-521.

Blais, M., Lévy, J., Bédard, I. et Corriveau, P. (2011). Régulation de l'homosexualité et homonégativité. Une analyse des facteurs économiques, culturels, juridiques et politiques à l'échelle internationale. Dans P. Corriveau et V. Daoust (dir.), La régulation sociale des minorités sexuelles. L'inquiétude de la différence (p. 227-253). Québec : Presses de l'Université du Québec.

Boellstorff, T. (2007). Queer studies in the house of anthropology. Annual Review of Anthropology, 36, 17-35.

Bourdieu, P. (1972). Esquisse d'une théorie de la pratique, précédé de trois études d'ethnologie kabyle. Genève : Librairie Droz.

Brotman, S. et Ryan, B. (2008). Une approche intersectionnelle des politiques et pratiques en matière de santé des gais, lesbiennes, bisexuels. Le cas des personnes bispirituelles au Canada. Dans S. Brotman et J. Lévy (dir.), Intersections. Cultures, sexualités et genres (p. 419-438). Ste-Foy : Presses de I'Université du Québec.

Bruce-Jones, E. et Itaborahy, L. (2011). Homophobie d'État. Une enquête mondiale sur les lois qui criminalisent la sexualité entre adultes consentants de même sexe. Un rapport de l'ILGA [Association Internationale des Lesbiennes, des Gays, des personnes Bisexuelles, Trans et Intersexuelles] [En ligne]. http://old.ilga.org/Statehomophobia/ILGA_Homophobie_Etat_2011.pdf

Butler, J. (1990). Gender trouble. Feminism and the subversion of identity. New York : Routledge.

Butler, J. (2007). Le récit de soi. Paris : Presses Universitaires de France.

Cantú, L. (2005). Well-founded fear: political asylum and the boundaries of sexual identity at the U. S.-Mexico borderlands. Dans E. Luibhéid et L. Cantú (dir.), Queer migrations. Sexuality, U. S. citizenship, and border crossings (p. 61-74). Minneapolis : University of Minnesota Press.

Chamberland, L. et Gagné, F. (2008). Parcours migratoires et identités gaies et lesbiennes. Dans S. Brotman et J. Lévy (dir.), Intersections. Cultures, sexualités et genres (p. 159-191). Ste-Foy : Presses de I'Université du Québec.

Conseil des droits de l'homme des Nations Unies (2011). Droits de l'homme, orientation sexuelle et identité de genre. Assemblée générale, XVII session. Récupéré sur le site Sexuality Policy Watch : http://www.sxpolitics.org/?p=6377

Crépeau, F., Heckman, G., LaViolette, N. et Rehaag, S. (2009, 12 novembre). Réfugiés écartés : La protection des demandeurs d'asile n'est pas un acte de générosité, mais une obligation juridique du Canada. La Presse, p. A22.

Da Cunha, P. (2003). Droit et récit. Ste-Foy : Les Presses de l’Université Laval. 
Delphy, C. (1991). Penser le genre : quels problèmes? Dans M.-C. Hurtig, M. Kail et H. Rouch (dir.), Sexe et genre : de la hiérarchie entre les sexes (p. 89-101). Paris : Presses du Centre national de la recherche scientifique.

Dembour, M.-B. (2001). Following the movement of a pendulum: between universalism and relativism. Dans J. Cowan, M.-B. Dembour et R. Wilson (dir.), Culture and rights. Anthropological perspectives (p. 56-79). Cambridge : Cambridge University Press.

Diallo, L. et Lafrenière, G. (2007). Intervenir auprès des survivants de guerre, de torture et de violence organisée : compte-rendu d'un projet de recherche entre l'Université Wilfrid Laurier et le Centre de santé communautaire de Hamilton et Niagara. Reflets, revue d'intervention sociale et communautaire, 13(1), 41-77.

Engel, P. (2005). Faut-il croire ce qu'on nous dit? Philosophie, 88, 58-71.

Fassin, D. (2004). Le corps exposé. Essai d’économie morale de l'illégitimité. Dans D. Fassin et D. Memmi (dir.), Le gouvernement des corps (p. 237-266). Paris : Éditions de l’École des Hautes Études en Sciences Sociales.

Fassin, E. (2003). Hétérosexisme. Dans L.-G. Tin (dir.), Dictionnaire de l'homophobie (p. 207-211). Paris : Presses Universitaires de France.

Geertz, C. (2000). Local knowledge: fact and law in comparative perspective. Dans Local knowledge. Further essays in interpretive anthropology (nouvelle éd., p. 167-236). New York, NJ : Basic Books.

Hastrup, K. (2003). Violence, suffering and human rights. Anthropological reflections. Anthropological Theory, 3, 309-323.

Hastrup, K. (2003b). Representing the common good. The limits of legal language. Dans R. Wilson et J. Mitchell (dir.), Human rights in global perspective. Anthropological studies of rights, claims and entitlements (p. 1632). Londres : Routledge.

Haut Commissariat des Nations Unies pour les réfugiés. (2008). Principes directeurs sur la protection internationale : La persécution liée au genre dans le cadre de l'article 1A (2) de la Convention de 1951 et/ou son Protocole de 1967 relatif au Statut des réfugiés. Récupéré sur le site du Haut Comissariat : http://www.unhcr.fr/4ad2f7f61d.pdf

Heinze, E. (2002). Sexual orientation and international law: a study in the manufacture of cross-cultural «sensitivity». Dans K. Hastrup et G. Ulrich (dir.), Discrimination and Toleration: new perspectives (p. 205-227). La Haye : Martinus Nijhoff.

Jenicek, A., Wong, A. et Lee, E. (2009). Dangerous shortcuts: representations of sexual minority refugees in the post-9/11 canadian press. Canadian Journal of Communication, 34(4), 635-658.

Jimenez, M. (2004, 24 avril). Gay refugee claimants seeking haven in Canada. The Globe and Mail. Récupéré sur le site de Sodomy Laws : http://www.glapn.org/sodomylaws/world/canada/canews020.htm

Johnson, P. et Henderson, M. (2005). Introduction: queering black studies/ «quaring» queer studies. Dans P. Johnson et M. Henderson (dir.), Black queer studies. A critical anthology (p. 1-17). Durham, NC : Duke University Press.

Jordan, S. (2011). Un/Settling: a critical ethnographic inquiry into settlement by refugees making claims based on sexual orientation and gender identity (thèse de doctorat, University of British Columbia, Vancouver, Canada). Récupéré sur le site de l’UBC à : https://circle.ubc.ca/handle/2429/30526

Kirmayer, L. (2003). Failures of imagination: the refugee's narrative in psychiatry. Anthropology and Medicine, $10(2), 167-185$.

Kleinman, A. et Kleinman, J. (1997). The appeal of experience; the dismay of images: cultural appropriations of suffering in our times. Dans A. Kleinman, V. Das et M. Lock (dir.), Social suffering (p. 1-23). Berkeley, CA : University of California Press.

Lacombe, P. (2008). Les identités sexuées et le «troisième sexe » à Tahiti. Les cahiers du genre, 45, $177-197$. 
Lacroix, M. (2004). Canadian refugee policy and the social construction of the refuge claimant subjectivity: understanding refugeeness. Journal of Refugee Studies, 17(2), 147-165.

LaViolette, N. (1997). The immutable refugees: sexual orientation in Canada (A. G.) v. Ward. University of Toronto Faculty of Law Review, 55(1), 1-41.

Lee, E. et Brotman, S. (2011). Identity, refugeeness, belonging: experiences of sexual minority refugees in Canada. Canadian Review of Sociology / Revue canadienne de sociologie, 48(3), 241-274.

Lee, E. (2009). Visualizing the margins: the experiences of queer people of colour (mémoire de maîtrise, McGill University, Montréal, Canada). Récupéré sur le site de l'Université : http://digitool.library.mcgill.ca/R/?func=dbin-jump-full\&object_id=67014\&local_base=GEN01-MCG02

Les Principes de Jogjakarta. (2007, mars). Principes sur l'application de la législation internationale des droits humains en matière d'orientation sexuelle et d'identité de genre. Récupéré sur le site des Principes : http://www.yogyakartaprinciples.org/principles_fr.pdf

Lidstone, R. (2006). Refugee queerings: sexuality, identity and place in canadian refugee determination (mémoire de maîtrise, Simon Fraser University, Vancouver, Canada). Récupéré du site de la bibliothèque de l’Université : http://ir.lib.sfu.ca

Loi canadienne sur l'immigration et la protection des réfugiés, LC 2001, c 27. (2011, 30 juin). Récupéré sur le site de I'Institut canadien d'information juridique : http://www.canlii.org/fr/ca/legis/lois/lc-2001-c-27/derniere/lc2001-c-27.html

Luibhéid, E. (2005). Introduction. Queering migration and citizenship. Dans E. Luibhéid et L. Cantú (dir.), Queer migrations. Sexuality, U. S. citizenship, and border crossings (p. ix-xlvi). Minneapolis : University of Minnesota Press.

Luibhéid, E. (2002). Looking like a lesbian. Sexual monitoring at the US - Mexico Border. Dans E. Luibhéid (dir.), Entry denied. Controlling sexuality at the border (p. 77-101). Minneapolis : University of Minnesota Press.

Mayer, T. (dir.) (2000). Gender ironies of nationalism: Sexing the nation. Londres : Routledge.

McGhee, D. (2003). Queer strangers: lesbians and gay refugees. Feminist Review, 73, 145-147.

McGhee, D. (2000). Assessing homosexuality: truth, evidence and the legal practices in determining refugee statusthe case of Ioan Vacriu. Body and Society, 6(1), 29-50.

Millbank, J. (2005). A preoccupation with perversion: the British response to refugee claims on the basis of sexual orientation, 1989-2003. Social Legal Studies, 14(1), 115-138.

Miller, A. M. (2005). Gay enough: some tensions in seeking the grant of asylum and protecting global sexual diversity. Dans B. Epps, K. Valens et B. Johnson Gonzales (dir.), Passing lines. Sexuality and immigration (p. 137-189). Cambridge, MA : Harvard University Press.

Murray, D. (2009). Homophobias. Lost and loathing across time and space. Durham, NC: Duke University Press.

Muzaffar, C. (1999). From human rights to human dignity. Dans P. Van Ness (dir.), Debating Human Rights (p. 2531). London : Routledge.

Nadeau, C. (2010). Contre Harper. Bref traité philosophique sur la révolution conservatrice. Montréal : Éditions du Boréal.

Nanda, S. (1997). Neither man nor woman: The Hijras of India. Dans C. Brettell et C. Sargent (dir.), Gender in crosscultural perspective ( $2^{\mathrm{e}}$ éd.) (p. 198-201). Upper Saddle, NJ : Prentice-Hall.

Naqvi, N. et Mujtaba, H. (1997). Two Baluchi Buggas, a Sindhi Zenana, and the status of Hijras in contemporary Pakistan. Dans S. Murray et W. Roscoe (dir.), Islamic homosexualities. Culture, history, and literature (p. $262-$ 266). New York, NJ : New York University Press.

Alterstice - Revue Internationale de la Recherche Interculturelle, vol. 1, $n^{\circ} 2$ 
Office québécois de la langue française (2005). Allosexuel; Allosexuelle. Grand dictionnaire terminologique [En ligne]. http://www.granddictionnaire.com/BTML/FRA/r_Motclef/index800_1.asp

Rainbow Refugee Committee. (2010). Le Rainbow Refugee Committee réagit au projet de réforme de la loi sur les réfugiés (projet de loi C-11) : effets des modifications proposées à la Loi sur l'immigration et la protection des réfugiés sur les demandeurs d'asile ayant été persécutés pour cause d'orientation sexuelle et d'identité sexuelle (OSIS) (mémoire présenté au Comité permanent de la Citoyenneté et de l'Immigration). Récupéré sur le site du Parlement du Canada :

http://www.parl.gc.ca/HousePublications/Publication.aspx ?Docld=4564195\&Language=F\&Mode=1\&Parl=40 \&Ses $=3$

Ramanathan, E. (1996). Queer cases: a comparative analysis of global sexual orientation-based asylum. Georgetown Immigration Law Journal, 11(1), 1-44.

Rehaag, S. (2011). 2011 Refugee claim data and IRB member recognition rates. Fichier 3, « Outcomes by board member (alphabetical) », récupéré du site du Conseil canadien pour les réfugiés : http://ccrweb.ca/fr/2011refugee-claim-data

Rehaag, S. (2008). Patrolling the borders of sexual orientation: Bisexual refugee claimants in Canada. McGill Law Journal/Revue de droit de McGill, 53(1), 59-102.

Renault, E. (2004). Le mépris social. Ethique et politique de la reconnaissance. Paris : Éditions du passant.

Rousseau, C., Crépeau, F., Foxen, P. et Houle, F. (2002). The complexity of determining refugeehood : a multidisciplinary analysis of the decision-making process of the canadian immigration and refugee board. Journal of Refugee Studies, 15(1), 43-70.

Rousseau, C. (2000). Les réfugiés à notre porte : violence organisée et souffrance sociale. Criminologie, 33(1), 185201.

Saillant, F. (2011). Droits humains et témoignages : l'épreuve de la culture. Alterstice, 1(2), 3-8.

Shamash, V. (1998). Being branché: a story of refugee advocacy and networking in Montréal and in cyberspace (mémoire de maîtrise, Université Concordia, Montréal, Canada). Récupéré sur le site de l’Université à http://spectrum.library.concordia.ca/536/

Smith, M. (2008). Identités Queer. Diaspora et organisation ethnoculturelle et transnationale des lesbiennes et des gais à Toronto. Dans S. Brotman et J. Lévy (dir.), Intersections. Cultures, sexualités et genres (p. 219-243). Ste-Foy : Presses de l’Université du Québec.

Stychin, C. (2004). Same-sex sexualities and the globalization of human rights discourse. McGill Law Journal/Revue de droit de McGill, 49, 951-968.

Warner, M. (dir.). (1994). Fear of a queer planet: queer politics and social theory. Minneapolis : University of Minnesota Press.

Welzer-Lang, D., Dutey, P. et Dorais, M. (dir.). (1994). La peur de l'autre en soi, du sexisme à l'homophobie. Montréal : VLB.

Whitaker, B. (2006). Unspeakable love: gay and lesbian life in the Middle East. Berkeley, CA : University of California Press.

Wilson, R. (1997). Representing human rights violations: social contexts and subjectivities. Dans R. Wilson (dir.), Human rights, culture and context. Anthropological perspectives (p. 134-160). Londres : Pluto Press.

Wintemute, R. (1995). Sexual orientation and human rights. The United States Constitution, the European Convention and The Canadian Charter. Oxford : Clarendon Press.

Yoshino, K. (2000). The epistemic contract of bisexual erasure. Stanford Law Review, 52(2), 353-461. 